\title{
Laboratory Instruments Management System Based on IOT
}

\author{
Zeliang Liu ${ }^{a}$, Baohai Yang ${ }^{b}$ and Ting Lu ${ }^{c}$ \\ School of Electronic Engineering, Jiujiang University, Jiujiang, Jiangxi, China \\ aaptblaze@163.com, byangbaohai@sohu.com, c luting524@163.com
}

Keywords: IOT; RFID; Zigbee

Abstract. Most colleges and universities have established an open lab, but there are two major problems, one is uncertain identity into the laboratory personnel, and the other is the safety of laboratory instruments. Using RFID and Zigbee technology, this paper gives the design of laboratory instruments management system based on Internet of Things, which conducted a fairly comprehensive test. Test results show that, the system had low hardware cost, achieved the identification, instrument positioning, license management and other functions, facilitated the management of laboratory equipment and personnel management.

\section{Introduction}

Things are all kinds of information sensing devices, such as a variety of devices and Internet radio frequency identification devices, infrared sensors, global positioning systems, laser scanners, etc. combine to form a vast network of all the objects connected with the network identification and management, it is the next generation of IT technology into full use in all walks of life, then things will integrate with the existing Internet, human society and the physical system integration [1] .

In recent years, with the demand for university teaching and teaching reform, more and more colleges and universities have established an open laboratory, teaching requirements preclude new teaching methods courses take reasonable real integration, project and so that the theory of teaching go into the lab, students learn by doing. Through research, found that an open laboratory construction and management primarily in the following two main problems [2] [3]: (1) laboratory teaching management is still in its traditional management stage, students have to enter the laboratory experimental projects operating practice, which makes the students' ability can not be fully exercise, while the lab is a large waste of resources. (2) As students on the use of experimental equipment can not track record, easily lead to equipment damage, lost and unable to be held responsible, in turn making the open experimental teaching can not promote a large range. Therefore, intelligent design a laboratory management system has a strong practical significance.

As the core of a new generation of information technology, networking is the integration of various technologies from the new technology system [4] , it will be based on the Internet any time, any place of communication and connection between people, extended to any time and any place and objects, object interaction and connections between things. Things architecture can be divided into "back-end centralized" and "front-end distributed" types [5-6]. Use networking technology can provide people with intelligent services, intelligent identification, positioning traceability, on-line monitoring, remote control and other functions. After analysis, the two major problems currently can be resolved which in Open University laboratory construction and management of existing networking technology.

\section{System solutions}

Currently, most universities have perfected the network system, a variety of wired and wireless networks across classrooms, dormitories, laboratories, etc., and have been widely used throughout the campus student campus card (RFID), therefore, the use of radio frequency identification system that can facilitate the realization of human and material laboratory management. The management system uses the campus card system authentication platform, the campus card authentication function, laboratory access control management, student attendance records management, security 
management circuit laboratory, laboratory equipment management combine to achieve the students through campus card online booking of experimental equipment, the administrator online approval and authorization, allowing students to swipe into the appropriate laboratory, and automatically recover the license after the experiment, the experiment time, frequency, data is automatically recorded by the system, and statistical analysis. Upon completion of the system development can take advantage of existing teaching resources to ensure the utilization of laboratory and equipment, student attendance may, laboratory usage, device usage data to provide support, while the use of laboratory conditions and utilization do quantified evaluation.

This system use RFID technology and Zigbee technology. RFID (Radio Frequency Identification) is the use of sensors, or electromagnetic means to transmit electromagnetic waves, non-contact bidirectional communication is completed, an automatic identification technology to obtain the relevant data [7-8]. ZigBee is a short-range, low-cost, low-power, low-complexity wireless networking technology, wireless sensor network applications in the field of potential development [9].

Laboratory instruments are generally expensive, but also teaching the required necessities, lost equipment, or equipment can not get it back in time to use, will result in economic losses and teaching inconvenient. Custody instrument consists of two aspects: first, the position at which the instrument; second is, who use the instrument. Zigbee technology can be used to locate the child nodes, so it can be used to locate the instrument in the laboratory Zigbee which position, and through the Zigbee network to the control center to send the instrument usage, to accept control commands issued by the control center. RFID technology can identify the person's identity, the instrument can be achieved by using a person's identity authentication to determine whether to authorize people to start using the instrument, and you can record who used the instrument, the instrument can be traced to the use of history, so that the instrument can also be damaged retrospective responsible. This system contains Personnel authentication, access management, student attendance records management, laboratory circuit management and laboratory Instruments management, as shown in Figure 1.

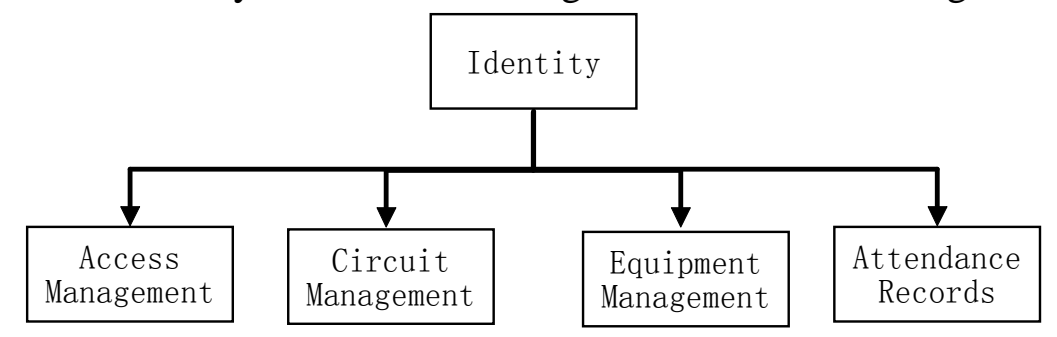

Fig.1. Block diagram of laboratory instruments management system

Access Control: Access to the laboratory personnel to the campus card as identification, real-time recording system will be out of time, personal information and other information.

Student Attendance Records Management: When a student out of the lab, in front of the reading device can read the user's identity through electronic tags, each person's attendance and tardiness and other conditions in real time into the time and attendance management repository.

Lab Circuits Safety Management: after personnel who come in lab using the card, laboratory equipment supply automatically turn on, the system begins recording experimental operation time, when person leave the laboratory, laboratory equipment auto power off.

Laboratory equipment management: monitoring and management center monitoring equipment storage state laboratory equipment, equipment purchase and run time.

\section{Design and implementation}

The hardware circuit design used in the main power supply board, RFID reader module, a wireless node module and relay control module. (1) Power board: it is necessary to achieve a wireless connection with the RFID reader module node module, but also supply the system power which convert AC into DC. (2) RFID reader module: on campus card identification, location and 
identification equipment testing. The main chip of the System is TRF7960. (3) Wireless node modules: mainly composed of RF microcontroller, MCU is CC2530 of TI Company, 2.4G carrier frequency, rod antenna. (4) Relay Control Module: The main issue commands to control the working status of the relay by the CC2530.

The TRF7960 is an integrated analog front end and data-framing system for a 13.56-MHz RFID reader system. Built-in programming options make it suitable for a wide range of applications for proximity and vicinity RFID systems. The reader is configured by selecting the desired protocol in the control registers. Direct access to all control registers allows fine tuning of various reader parameters as needed. A parallel or serial interface can be implemented for communication between the MCU and reader. Transmit and receive functions use internal encoders and decoders with a 12-byte FIFO register.

The CC2530 is a true system-on-chip (SOC) solution for IEEE 802.15.4, Zigbee and RF4CE applications. It enables robust network nodes to be built with very low total bill-of-material costs. The CC2530 combines the excellent performance of a leading RF transceiver with an industry-standard enhanced $8051 \mathrm{MCU}$, in-system programmable flash memory, 8-KBRAM, and many other powerful features. The CC2530 comes in four different flash versions: CC2530F32/64/128/256, with32/64/128/256KB of flash memory, respectively. The CC2530 has various operating modes, making it highly suited for systems where ultralow power consumption is required. Short transition times between operating modes further ensure low energy consumption. Combined with the industry-leading and golden-unit-status ZigBee protocol stack (Z-Stack ${ }^{\mathrm{TM}}$ ) from Texas Instruments, the CC2530F256 provides a robust and complete ZigBee solution.

Since CC2530 chip provides a complete protocol to find programs, routines rich, easy to develop, and is a built-in MCU SOC chip. The system uses Zigbee CC2530 as the core chip. NXP's RF reader chip RC522 and TI's reader chip TRF7960 are common, RC522 been widely used which has cheap price, affordable, and mature technology. TRF7960 RF chip will automatically detect the reader into the label range, TRF7960 can support different protocol configuration registers, mainly supported protocols: ISO15693, ISO1443A / B and TI's Tag-it protocol [10-12]. This system uses the TRF7960. Laboratory instrument's status is controlled by relays. This is the overall design diagram of the system as shown in Figure 2.

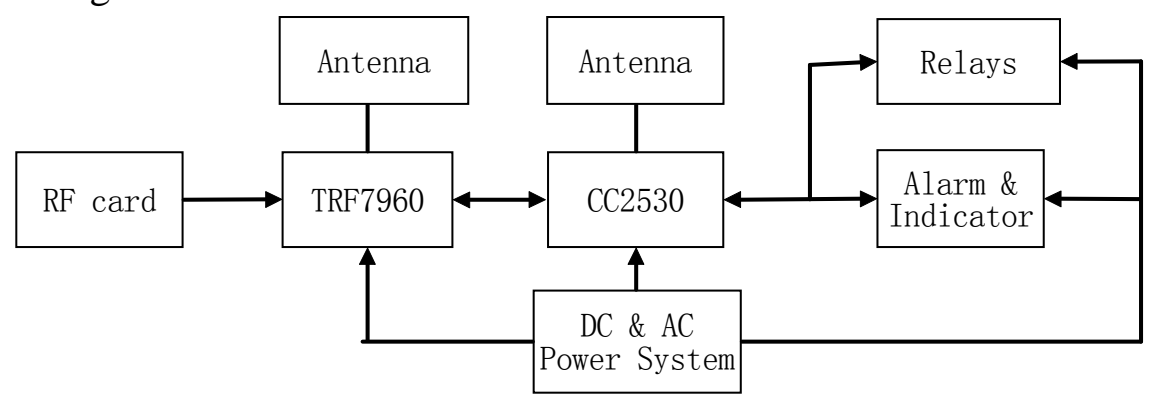

Fig.2. Structure of laboratory instruments management

Software design process is divided into four parts, namely a terminal program, router program, the coordinator program and host computer service program. These programs use a complex Zigbee protocol stack and QT programming techniques, program structure is more complex. Terminal program detect whether the RFID card of the system can be read, and report their location to master computer every interval. When reading to effectively put RFID card information sent to the PC card for authentication. When the laboratory after receipt of authorization to open the door and give the experimental apparatus is energized, the instrument starts counting time. If the end user card, authorize the use of the termination, the system will automatically cut off the power to the instrument. The flow diagram of terminal program is shown in Figure 3. 


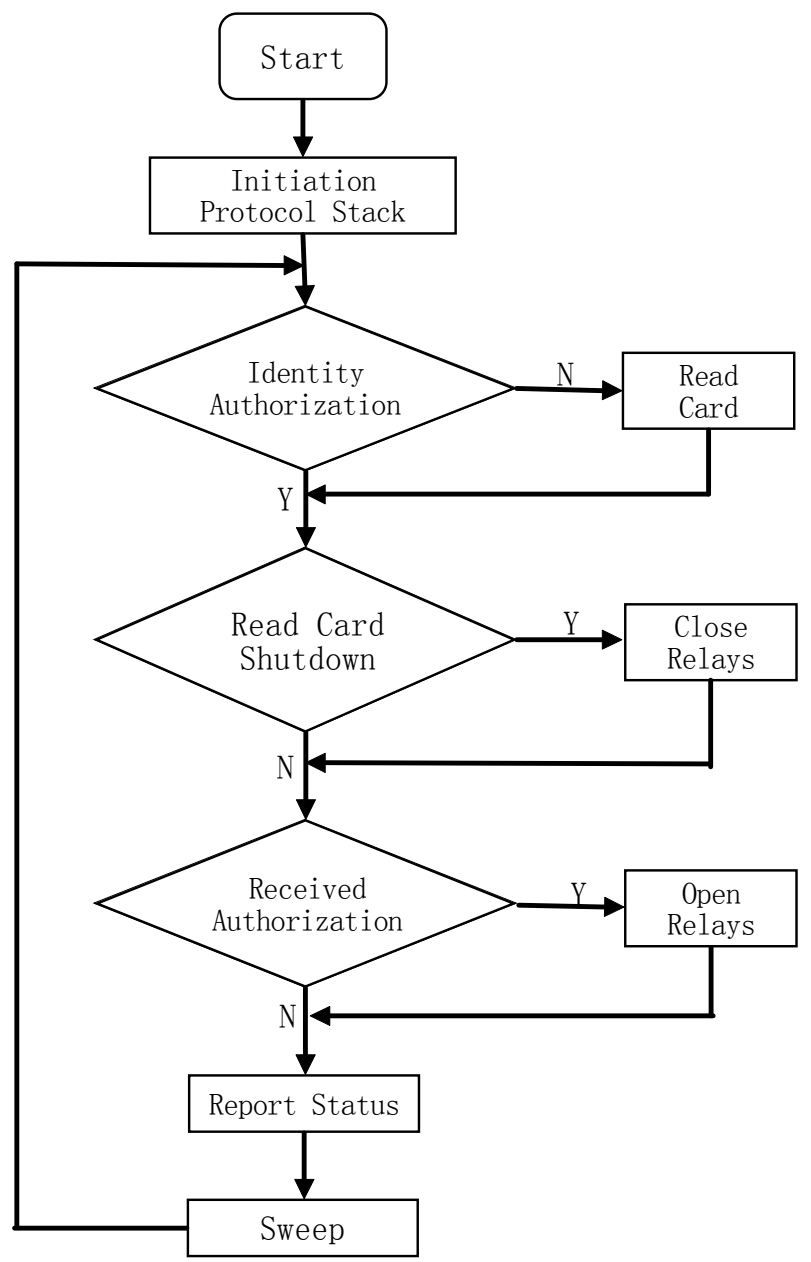

Fig.3. Flowchart of the terminal program

Router mainly completes the forwarding function, which is the default function of protocol stack. Another function is to receive data child nodes, additional information on their own, and forwards to the host computer. Coordinator receives data from the Zigbee network, and forwards it to the host computer by serial port. Host computer Opens serial port, communicates with the coordinator, gets data from the Zigbee network and processes data, which will be displayed on the window interface.

\section{Design Verification}

Finally, the system was tested, including interface testing, functional testing, and abnormal tests. Interface test is confirmed interaction between the user and the software, the goal is to ensure that the user interface provides the user with the appropriate access and browsing operations. In addition, make sure the software interface testing features inside the object meets expectations, and follow industry standards. Functional testing is to validate the various features of the product, according to functional requirements itemized tests to check whether the user needs the product functionality. Abnormal test is done by a variety of limits and beyond the standard input data, the system robustness test, the test system in case of failure, is able to automatically restore or ignore the failure to continue to run. To make the system has good robustness, requires the designer to do system design must be careful and detailed, with particular attention to the system properly handle exceptions. After testing, the system user-friendly, with the identification, access control, student attendance records management, security management circuit laboratory, laboratory equipment management and other functions, to good effect, the main operation interface shown in Figure 4. 


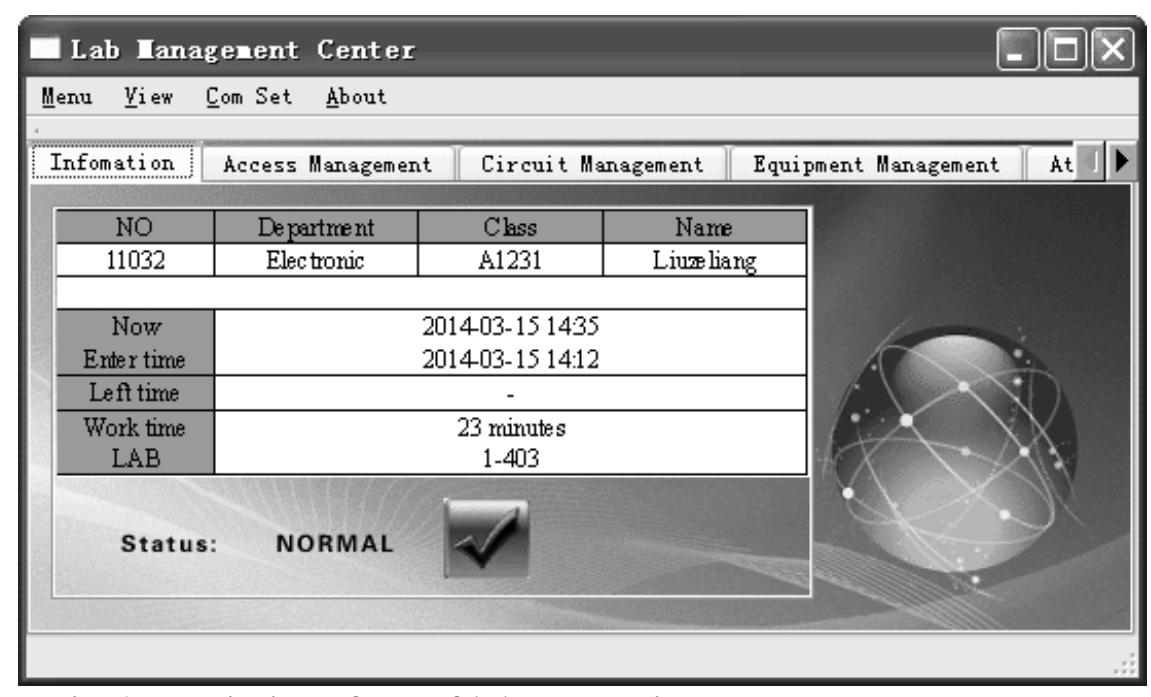

Fig.4. Main interface of laboratory instruments management

\section{Conclusion}

Most colleges and universities have set up an open laboratory. The identity of uncertainty into the laboratory personnel not only results the safety hazard to laboratory equipments, but also increase the workload of laboratory staff. This paper introduces RFID technology and Zigbee technology, proposed management system based on the Internet of things. Laboratory instruments management system which combines laboratory equipment management, student experiments curriculum management, to school uniform use of the card together, allows students to use instruments by credit card, achieves authorization management of instrument, and locates instruments to facilitate the laboratory equipment management, the students to do experiments, to facilitate teachers to teaching management. The low hardware cost and the practicality are the most values.

\section{Acknowledgements}

This work was financially supported by the Educational Commission of Jiangxi Province, China (GJJ14743), Scientific Research Fund of Jiujiang science and technology bureau (64) and Natural Science Foundation of Jiujiang University (2014KJYB002,2014KJYB006).

\section{References}

[1] Wang CaiFeng and Wang Yanli, “IOT technology application in university campus,” Modern computer, Issue 4, pp. 56-57, 2012.

[2] Zhang Ting and Wang Yuan, "Laboratory management system design and implementation based on B/S structure,” Computer Development \& Applications,2010.

[3] Yan Lihui, "A brief analysis of the open experimental teaching and laboratory management system,” Scientific Friends, Issue 4, 2012.

[4] Liu Qiang, Cui Li and Chen Haiming, "IOT key technologies and applications,” Computer Science, vol. 37, Issue 6, pp. 1-4, 10, 2010.

[5] Chen Haiming, Cui Li and Xie Kaibin, "A Comparative Study of the structure and implementation of the system of things,”Journal of Computers, vol. 36, Issue 1, pp. 168 -188, 2013.

[6] Wang Jin, "Laboratory management technology based on IOT," Computer Knowledge and Technology, vol. 6, No 21, pp. 5741-574, 2010. 
[7] Michele Zorzi, University of Padova, From Today's Internet of Things to a Future Internet of Things: a Wireless and Mobility Related View [J], IEEE wireless Communications, 2010, 1536-1284:44-51.

[8] Wang Baoyun, "Research Review Things,” Journal of Electronic Measurement and Instrument, Issue 12, pp. 1-5, 2009.

[9] ZigBee Alliance. http://www.zigbee.org/en/events/documents.

Architecture Overview

$[\mathrm{EB} / \mathrm{OL}]$

[10]Chang Guoquan, Wei Shengli and Xie Lijiang, "Multi-protocol RF card reader design Based on TRF7960,” Microcontroller and Embedded Systems[J], vol. 12, No7, pp. 76-80, 2010.

[11]Using the SPI Interface with TRF7960 [EB/OE]. http://www.ti.com, 2009.4

[12]Firmware Description of the TI TRF796x Evaluation Module [EB/OE]. http://www.ti.com, 2009.3 